\title{
Necrotizing fasciitis of Face, an untold Mystery
}

\author{
Dr. Swapnil Moghe ${ }^{1}$,Dr. M.K. Gupta ${ }^{2}$, Dr. Anjali Moghe ${ }^{3}$, Dr. Ajay Pillai ${ }^{4}$, Dr. \\ Nikit Agrawal ${ }^{5}$,Dr. Saksham Nahar ${ }^{6}$. \\ ${ }^{I}$ (Reader, Department of Oral \& Maxillofacial Surgery, Peoples Dental Academy, Peoples University, Bhopal, \\ Madhya Pradesh, India.) \\ ${ }^{2}$ (Professor, Dean \& H.O.D., Department of Oral \& Maxillofacial Surgery, Peoples Dental Academy, Peoples \\ University, Bhopal, Madhya Pradesh, India.) \\ ${ }^{3}$ (Ex. Lecturer, Department of Oral Medicine \& Radiology, Peoples Dental Academy, Peoples University, \\ Bhopal, Madhya Pradesh, India.) \\ ${ }^{4}$ ( Reader, Department of Oral \& Maxillofacial Surgery, Peoples Dental Academy, Bhopal, India.) \\ 5 (Post graduate Student, Department of Oral \& Maxillofacial Surgery, Peoples Dental Academy, Bhopal, \\ India.) \\ ${ }^{6}$ (Post graduate Student, Department of Oral \& Maxillofacial Surgery, Peoples Dental Academy, Bhopal, \\ India.)
}

Abstract: Necrotizing fasciitis (NF) is a rare infection of the facial planes and it is less common in head and neck, because of the rarity and higher vascularity in the region. We report a case of necrotizing fasciitis in a 67year-old man, arising from a dental infection treated successfully by early diagnosis, prompt surgical management, antibiotic therapy and adjunctive hyperbaric oxygen (HBO) therapy.

It is associated with a high mortality and results in severe disfigurement of the face, posing challenging reconstructive problems. The authors present their experience on the comprehensive management of NF of face Key Words: with severe disfigurement affecting a 67 year old male patient.

\section{Introduction}

Necrotizing fasciitis (NF) is an extremely rare, usually multi microbial infection of the skin and subcutaneous tissues, characterized by rapid invasion of soft connective tissue ${ }^{1}$. Patients with immunocompromised status such as diabetes mellitus, cancer, alcoholism, vascular insufficiencies, organ transplants, HIV or neutropenia are prone to this type of infection ${ }^{2}$.

NF is a rare infection usually caused by $\beta$-haemolytic Streptococcus. The condition was first described by Meleny (1924) in China ${ }^{3}$, it is typified by cutaneous necrosis, suppurative fasciitis, thrombosis of small blood vessels in the subcutaneous tissue and extreme systemic toxicity ${ }^{4}$.

A 67-years-old male patient was referred to our department with a history of Swelling \& with overlying necrotic skin on the lower part of left cheek of 8 days duration with associated pus discharge and toothache in the left side of lower jaw. This was preceded by a diffuse swelling over left side of face that had lasted 7 days before bursting (Figure 1). The patient was administrated Cap. Amoxycillin $500 \mathrm{mg}$ 8th hourly by the referring doctor for 5 days. The patient was not on any medication at the time of presentation.

Intraorally, pericoronitis was noticed in relation to partially erupted left mandibular third molar which was perceived to be the source of infection. Incision \& drainage was done (Figure2). Local examination revealed necrotic tissue measuring about $2 \times 3 \mathrm{cms}$, beneath of which was slough, the surrounding skin was blackish and edges showed slight undermining (Figure 3). There was evidence of disintegration of the subcutaneous fat. The muscular fascia was necrotic and peeled off easily during dressing (Figure 4). There was a distinct putrid odour when the patient first presented. Medical/ family/social history was unremarkable. 


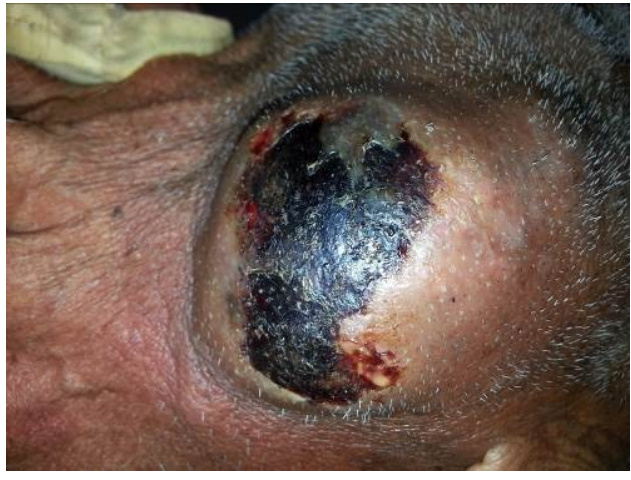

Figure 1: Preoperative picture Showing necrosis of overlying skin.

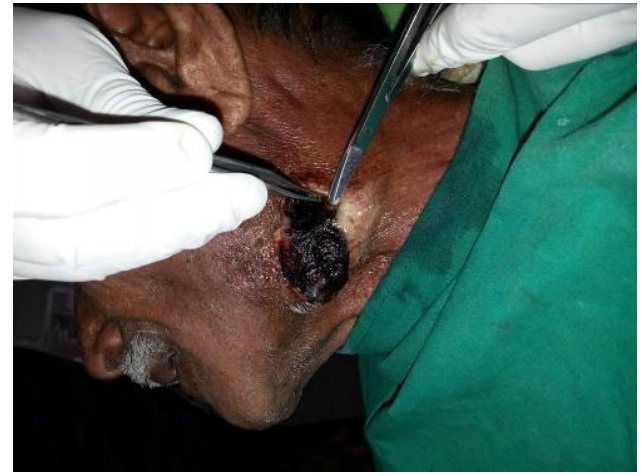

Figure 3: Removal of Overlying necrotic tissue.

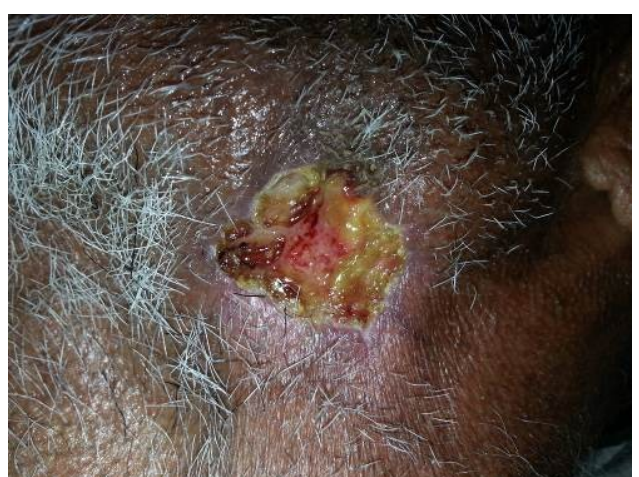

Figure 5: Secondary healing at operated site.

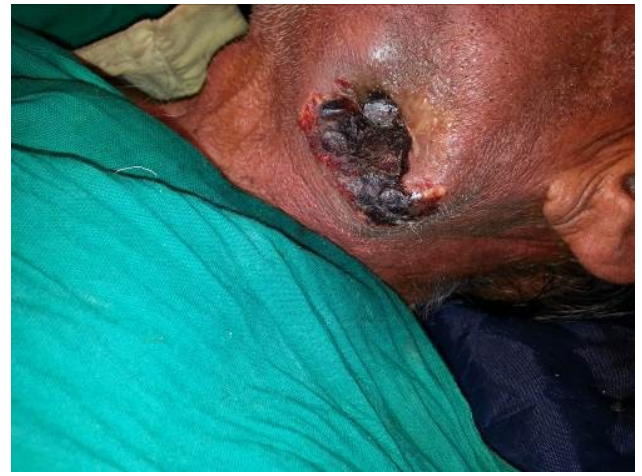

Figure 2: Clinical picture after Incision \& Drainage.

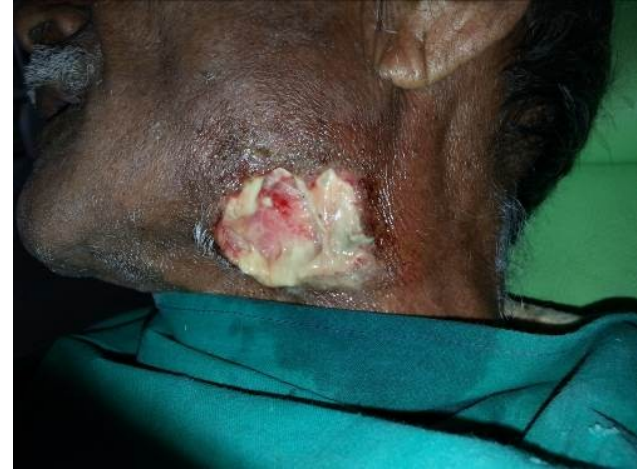

Figure 4: Raw area after removal of necrotic tissue.

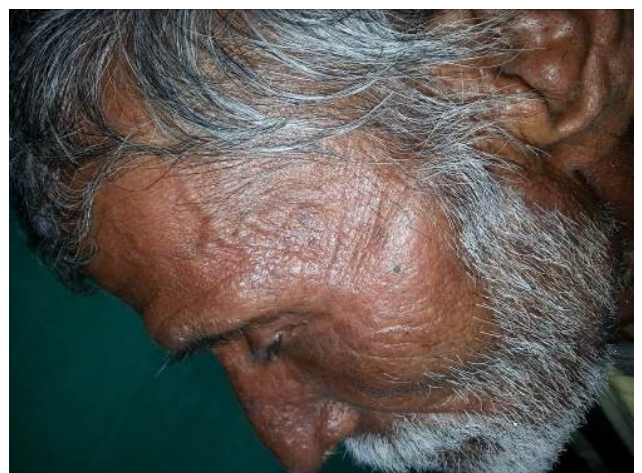

Figure 6: Postoperative picture with hair growth $\&$ good healing.

\section{Investigations}

Laboratory investigations were within normal limits. A $\beta$-haemolytic streptococcus was isolated from pus culture which was highly sensitive to cephalosporin and moderately sensitive to amoxycillin. OPG was done suggestive of involvement of left mandibular third molar.

\section{Treatment}

Amoxycillin $500 \mathrm{mg}$, 8th hourly was administered at the time of admission and withdrawn on receipt of the culture and sensitivity report following which, Inj. Ceftriaxone $1 \mathrm{gm}, 12$ th hourly was administered for a period of 8 days. Multivitamins and high protein diet were provided.

Following vigorous debridement and regular dressings with saline and $\mathrm{H}_{2} \mathrm{O}_{2}$, initially the dressings were changed daily for the first 5 days and then as the soakage reduced, the frequency was reduced to alternate 
days with light dressing. The wound became better but leaving defect of approximately $1 \times 1 \mathrm{cms}$ in the neck. The defect was allowed to heal by secondary intention. The offending third molar tooth was extracted.

\section{Surgical Procedure}

Considering the age of patient, Incision $\&$ drainage was done $\&$ necrotic tissue was removed. We have decided to leave the defect raw $\&$ allow it to heal by secondary intention (figure 5).

\section{Outcome And Follow-Up}

Follow-up was done for 6 months and the result is good, as the patient was highly satisfied with the final outcome (figure 6).

\section{Discussion}

Wilson proposed the term NF in 1952. NF usually occurs in the perineum, lower limbs or abdominal wall following surgery or trauma, particularly in individuals with underlying systemic diseases such as diabetes mellitus, arteriosclerosis, chronic renal failure or malnutrition. NF is rare in the head and neck. An odontogenic infection is the most common cause followed by tonsillar and pharyngeal infections ${ }^{5}$. In most cases of NF, bacterial invasion occurs as a result of some precipitating event that produces a break in the epidermis.

During the early stages, pain can be the presenting symptom; cutaneous necrosis owing to thrombosis of nutrient vessels becomes apparent by the fourth or fifth day. Eventually, the necrotic tissue begins to separate by suppuration by the eighth day ${ }^{6}$. As the necrotizing process continues to spread, soft tissue components below and above the affected fascia may become involved. Complications such as airway obstruction, mediastinitis, pleural empyema, large vessel thrombosis and septic shock may be fatal.

Prompt and successful management of NF is by aggressive surgical intervention, intravenous widespectrum antibiotics, supportive therapy and oxygenation of the damaged tissues with a hyperbaric chamber.

Four factors that contribute significantly to morbidity and mortality of NF are (1) delayed treatment, often owing to difficulty in recognizing the condition; (2) inappropriate treatment; (3) host debilitation and (4) a poly-microbial infection ${ }^{7}$. The mortality rate for NF is significant, approaching $73 \%$ despite the treatment with appropriate antibiotics. A delay in diagnosis is speculated to be the most likely reason for the significant mortality. Adjunctive HBO therapy might decrease the mortality and limit the extent of debridement in patients with $\mathrm{NF}$, but the results have been conflicting.

HBO reinstates the defense against infection by increasing free radicals, which helps neutrophil mediated killing of some common bacteria. In addition, HBO therapy acts as a bactericide for certain anaerobes. HBO reduces the mortality, shortens the duration of hospitalization and decreases the required number of surgical debridement's.

\section{Acknowledgement:}

The authors would like to thank Dr. M.K. Gupta, Dr. Anjali Moghe, Mom \& Dad for their constant encouragement \& support. Without them this work would be unfinished.

\section{Learning points:}

1. NF is rare in young patients, if not recognized and unmanaged; it may lead to severe disfigurement. Its a rare entity in the facial region requiring reconstructive procedures.

2. The condition has a tendency to progress in a subcutaneous plane often not affecting the overlying skin, the surgeon has to pay attention to clearing necrotic tissue under the surrounding skin.

3. Aggressive surgical debridement of all involved tissue along with intravenous antibiotic therapy should be initiated before aerobic and anaerobic cultures are obtained.

\section{References}

[1]. Shimizu T, Tokuda Y. Necrotizing fasciitis. Inter Med 2010; 49: 1051-7.

[2]. Lanisnik B, Cizmarevic B. Necrotizing fasciitis of the head and neck: 34 cases of a single institution experience. Eur Arch Otorhinolaryngol 2010; 267:415-21.

[3]. Benavides etal, Necrotizing fasciitis of the face: a report of one successfully treated case. Otolaryngol Head Neck Surg 2003; 128:894-6.

[4]. Shindo etal, Necrotizing fasciitis of the face. Laryngoscope 1997; 107:1071 - 9 .

[5]. Ali MH, Zayed ME. Necrotizing fasciitis of the head and neck: report of three cases. Ann Saudi Med 1997; 17: 641-5.

[6]. Muto T, Sato K, Kanazawa M. Necrotizing fasciitis of the neck and chest: report of a case. Int J Oral Maxillofac Surg 1992; 21:236-8.

[7]. Edwards JD, Sadeghi N, Najam F, et al. Craniocervical necrotizing fasciitis of odontogenic origin with mediastinal extension. Ear Nose Throat J 2004; 83:579-82. 